\title{
The Effect of Body Composition and Physical Fitness Factors on Performance Prediction of Elite Male Trampolines
}

\author{
Izzet Uçan \\ Correspondence: Izzet Uçan, Faculty of Sport Sciences, Bayburt University, Bayburt, Turkey. \\ Received: February 1, 2018 \\ doi:10.11114/jets.v6i4a.3258 \\ Accepted: May 4, 2018 Online Published: May 4, 2018 \\ URL: https://doi.org/10.11114/jets.v6i4a.3258
}

\begin{abstract}
The main purpose of this study was the effect of body composition and physical fitness factors on performance prediction of elite male trampolines. So, 45 male athletes, who participated in the national trampoline team's preparation camps for participation at the 2014 Asian Games in four age categories included Children, Juniors, Adolescents and Seniors were selected using targeted sampling as subjects. Variables of study included 24 body composition indices and 20 physical fitness factors which were measured using standard procedures and instruments. Data were analyzed using Pearson correlation coefficient and multiple regression models at the 0.05 significance level. Results of study showed that, the relationships of the shoulder belt muscle strength $(r=0.294)$ with performance are statistically significant and in other cases, there was no significant relationships $(p>0.05)$. Results of study showed the relationships of the shoulder belt muscle strength $(r=0.294)$, aerobic power $(r=0.351)$, relative minimum anaerobic power $(r=0.256)$, with performance are statistically significant.and in other cases, observed relationships were not statistically significant $(p>0.05)$. Findings of this study suggest that, in the high competitive level of trampoline body composition indices do not have predictive role in preparation programs of elite male trampolines, but physical factors have predictive role in preparation programs of elite male trampolines.
\end{abstract}

Keywords: body composition, physical factors, trampoline, elite athlete

\section{Introduction}

Trampoline's competition has become one of the most important sports events in Asia and the Olympics, and has attracted the attention of champions in recent years. National teams of this field are regular and with long-term planning in the country and their national teams are represented in various international competitions. In the meantime, competitive success in this sport requires the development of physical and mental training programs for athletes, which, in turn, requires detailed information on the requirements of the field of trampoline and the importance of each. The factors involved in athletic performance should be studied scientifically and systematically. Unfortunately, despite the importance of the field of trampoline, very little attention has been paid by domestic and foreign researchers to this sport, and there is no accurate information about it. The results of extensive research in the field of championship sport show that the ideal sport performance in any sport is influenced by a combination of morphological, physiological, psychological and sociocultural factors (MacMillan 2007). Although it is worth exploring how to combine these factors into each and every field of sport separately and proprietary, however, due to the lack of research in the field of trampoline, and on the other hand, the close connection between the trampolines and gymnastics, scientific information reported in gymnastics. Studies conducted on gymnasts show that these athletes have special morphological, physiological and psychological needs.

For example, Douda et al. (2008) in their research on elite and amateur groups of gymnasts showed that the anthropometric components of $45 \%$ are involved in gymnastic performance.

In a recent study by Gomez-Landro et al. (2010), the species and body composition of the tropical women of the elite female elderly were studied. The results of this study showed that trampolines have different types of gymnasiums of different types, such that trampolines have less ectomorph in their physical form and are more endomorphic. This evidence can be a reason for different requirements of the discipline Trampolines and gymnastics. However, due to the lack of research in the field of trampoline, the current information on analyzing the needs of this field is in the speculations of experts, and definitive comments on it cannot be presented. Accordingly, the present research aims to determine and prioritize the needs of the trampoline field, which seeks to investigate the correlation of selected physical capabilities of elite male trampolines at different age ranges, including preterm infants, adolescents, young adults and adults, and to 
predict the performance of these athletes on the basis of physical fitness.

Dzhafarov \& Vasil'chuk (1987) examined the anthropometric characteristics of elite female gymnasts that gymnasts with a technical level of leveling (scores of about 10) had similar anthropometric profiles. Faria and Faria (1989) concluded that the association between anthropometric and physical characteristics of elite young men's gymnasts with competitive performance led to the conclusion that class 1 gymnasts were with a shorter stature, relative strength and absolute, higher muscle mass.

Lindner, Caine \& Johns (1991) in the study of physical and functional predictors by withdrawal competitive female gymnasts concluded that withdrawal gymnasts were older, taller and heavier, which were the reason for their higher strength, power, speed, and endurance. Discontinued gymnasts with less skinny physics/ectomorphic with less muscle had better recordings in most of the flexibility tests.

Claessens \& Lefevre (1998) in examining the morphological and functional characteristics as the foreclosure predictors in female gymnasts in Belgium, it was concluded that anthropometric variables and physical fitness are not an important factor in quitting the championship competitions And only age is a decisive factor that may be related to social and psychological factors.

Casenz et al. (1999) examined the role of anthropometric features in the performance of elite female gymnasts, and concluded that there was a moderate and inverse association between the score of gymnasts in the balance body and the thickness of the skin of the dorsal joint in China. There was a strong and inverse association between the overall performance of the gymnasts and the endomorphic component. These results suggest that gymnasts with lower subcutaneous fat and higher endomorphs have lower performance.

Ackland et al. (2000) examined the effect of body size growth on the four-month period on rotational performance in gymnasiums. Short gymnasts with higher strengths have more potential to implement skills that involve the whole body. They have it. Taller gymnasts, while capable of generating more power and angular velocities, are not as good as short-range gymnasts.

Grandjean, Taylor \& Weiner (2002) in examining the relationship of assurance in implementing and focusing on competitive performance in gymnasiums at the 2000 Olympics found that reliance on gymnastics implementation did not directly correlate with performance, but the linkage with the mediation of gymnastic focus was on the run.

Giorpopoulos et al. (2004) concluded that men and women gymnasts studied skeletal growth and artistic gymnastics in men's gymnasts compared to those with a taller height, lower lumbar bone age, higher body mass index, less body fat and age began to practice higher.

Rodriguez and Bral Di La Rosa (2006) in their study described the anthropometric profiles of male elite male gymnasts and concluded that the structure of the elite Argentine gymnasts has a biomechanical advantage in comparison with outstanding gymnasts is not higher and may interfere with their competitive performance.

Di Cagno et al (2008) considered the anthropometric characteristics of young and adult elite gymnasiums in Italian rhythmic gymnastics between 2002 and 2006. The effect of the time period is only significant on the width of the two shoulders and the pelvis. There was no significant difference between the 2002 and 2006 gymnasts' characteristics. These results indicate that the growth pattern of male gymnasts is the same as ordinary participants, with the difference that male gymnasts are mostly selected from the beginning with unique features.

In a study aimed at identifying factors affecting motor performance in amateur gymnastics with an emphasis on gender differences, the results of this study showed that height, length of the lower limbs and body fat were positively and moderately correlated with the mean of 0.40 to 0.6 with jump height and flight time in gymnastic skill tests, which indicates the importance of these factors in the gymnastics motion performance.

Gómez-Landero et al. (2010) studied the species and physical composition of the Spanish elite female gymnasts in the competition material of trampoline, it was concluded that trampolines have different physical types of artistic gymnasts, such that the trampolines of things has a lower electrophormity and an endomorphic.

Zuniga et al. (2011) examined the annual changes in anthropometric characteristics of high school female gymnasts in comparison with national norms and concluded that participation in gymnastics did not have a negative effect on the physical growth pattern.

Faria and Faria (2012) studied the relationship between the physical characteristics of young elite male gymnasts and their competitive performance. Based on the results, gymnasts of the first class had a shorter stature, more relative strength and absolute strength, more shoulder flexion, lower back and pelvic, higher muscle mass and lower percentage of fat. Lindner, Caine \& Johns (2013) examined the physical and functional predictors of competitive withdrawal in female gymnasiums. The results of the study showed that dropout gymnasts are older, taller and weigh heavier, which 
were the reason for their higher strength, power, speed and endurance. Discontinued gymnasts with less skinny physics/ ectomorphic with less muscle, had better recordings in most of the flexibility tests.

The exclusive gymnastic flexibility test was the only factor able to distinguish gymnasiums involved in competitions from gymnasiums. According to their observations, the researchers concluded that they were the only factor of age and social and psychological factors that distinguished gymnasts from competitive gymnasiums.

Hume et al (2014) studied the physical factors determining progress in rhythmic gymnastics. Based on the results, the best correlates of the progress of gymnasts were cumulative training time and training time $(\mathrm{r}=-0.530 .84)$. Also, age and muscle mass and body composition, flexibility, lower limb strength, visual-motor performance had significant relationships with the level of progression $(r=-0.29)$.

Dashti (2015) studied the relationship between anthropometric characteristics and selected physical fitness factors with the performance of gymnasts of Iranian male boys. The results of this study showed that variables such as height, length of two hands, fat and body weight without fat, finger power and power have a significant relationship with the performance of primary gymnasts. However, there was no significant relationship between the width of shoulder, hip width, hip length, percentage of fat and pelvic flexibility.

Douda et al. (2016) identified the physiological and anthropometric predictors of rhythmic gymnastics. The results showed anthropometric components of $45 \%$ of total variance, $12.8 \%$ flexibility, explosive power $9.2 \%$, aerobic power of $7.4 \%$, body dimensions of $6.8 \%$ and fueling $4.8 \%$. Anthropometric features $(r=0.5)$ and aerobic power $(r=0.49)$ had a significant correlation with performance. The results of regression analysis showed that hip circumference and body mass index explained $13.1 \%$ and $8.5 \%$ of variance, respectively. Therefore, anthropometric, aerobic, flexural and explosive power characteristics were reported as important determinants of successful performance.

Nowzuhari (2016) showed that the role of anthropometric features in the performance of elite gymnasts is $24.2 \%$. Despite the knowledge provided in the field of gymnastics, it seems that the requirements of the trampoline range are different in some sports-related factors than gymnastics. For example, trampolines may have a higher need for static and dynamic equilibrium than their gymnasts. Also, in a coordinated two-dimensional competition, athletes in this field will need more time to take photos and coordinate their movements.

\section{Materials and Methods}

The present research is considered as a practical one and in terms of the method it is a predictive correlation research.

\subsection{Society, Sample and Sampling Method}

The statistical population of the study was all male trampolines who were invited to the Islamic Republic of Iran gymnastics training camps in 2014 to participate in Asian Games 2014 and participated in competing selections within the campus. These include 48 athletes in six competitive age groups including L5, L6, L7, L8, which included 11, 12, 13, 14, $15-17$ and 18 years old.

In a more general division, 48 athletes were invited to the national team camps in four age groups (11 and 12 years old), adolescents (13 and 14 years of age), youth (15-17 years) and adults (18 years) (In each age group 12 participants). Due to the limited number of participants in the statistical population, non-random sampling method was used. As a result, the research sample was equal to the statistical society. However, 3 participants in camps were unable to complete measurements due to injury, which dropped out of the sample and the number of samples dropped to 45 . All participants, with full knowledge of the goals and implementation process, participated in this study with full satisfaction.

\subsection{Measuring Tools and Equipment}

In the present study, the following tools and equipment were used to measure the variables of the research:

1. Researcher-made questionnaire for collecting individual information of subjects

2. Digital scales with accuracy of $0.01 \mathrm{~kg}$ equipped with a precision 0.005 meter with SOEHNLE® marking Made in Germany to measure the height and weight of subjects

3. Anthropometric meter for Quick Medical ${ }^{\circledR}$ marker ANTTAPS US for measuring distance and lengths in different tests

4. Body composition analysis device with Zeus $+9.9+$ Swiss model for determining the body composition of subjects

5. Standard trunk flexural test for measuring the flexural strength of the front trunk

6. Refereeing Board and Rules of Arbitration of Gymnastics Federation to measure the performance of the subjects.

7. Horizontal stretching test for measuring muscle strength of shoulder belt in subjects

8. Abdominal examination on the Swedish ladder to measure the abdominal strength of the subjects 
9. Specific test of angle maintenance on the parallel to measure the muscular endurance of the subjects

10. Standard shoulder flexion (extension and flexion) tests for assessing the joint flexion of the subjects

11. Standard trunk flexural test for measuring the flexural strength of the front trunk

12. Standard trunk flexural test for measuring trunk flexion to the back of the subjects

13. Monarch Aerobic test on Treadmill with TechnoGym ${ }^{\circledR}$ Brand made in Italy to Test Aerobic Exercise

10. Vertical jump and jump tests to test the subjects' explosive power

11. Monarock's Tissue Test on a TechnoGym ${ }^{\circledR}$ Marker Bike made in Italy to measure the muscle strength of the upper body of the subjects

12. Argus test for 15 seconds to measure the muscle strength of the lower body of the subjects.

13. Zigzag test for 30 seconds to test the agility of the subjects

14. Test of the time of the photo-observation operation 8 directions for measuring the time of the photo-observation of the subjects

15. Test of the 8-way star movement for measuring the dynamic balance of the subjects

16. Arbitration Board and Arbitration Rules of Gymnastics Federation to assess the performance of the subjects

\section{Measurements}

\subsection{Personal Information}

Participants' individual information was comprised of eight questionnaires for determining age, competitive age, sporting history, competitive history, membership in the national team, weekly physical and psychological hours, and collecting history records, respectively, using a researcher-made questionnaire.

\subsection{Body Composition}

In this study, the body composition analysis was used to analyze body composition using the Mark $9.5+9.9$ model. Indicators used in this study included height, body mass, body mass index, intercostal water content, protein percentage, soluble mineral content, fat percentage and fat mass and muscle of the body. In calculating various indices were used.

\subsection{Competitive Performance in the Implementation of Trampolines}

In order to measure the level of performance of the participants, information about the selection of the camp that was carried out according to the laws of the Islamic gymnastics federation of the Islamic Republic was used. Based on this intra-camp competition, each participant will execute 20 trampoline motor skills and assess the quality of the performance by five national referees.

The range of scores provided by each referee is between 0 and 20 , which is based on the qualitative analysis parameters of skill implementation for each participant. Accordingly, the score for each participant's score is between 0 and 400. In calculating the performance score, at first, the minimum and maximum grades are eliminated from the five points given for each skill and the average of the three remaining scores is calculated.

Participants were also asked to respond to the questions with integrity and precision, given that there was no right or wrong answer in the questionnaires. At the end of the coordination session, the recommendations were provided by the researcher to the participants about the amount of sleep, nutrition and rest. Then, after coordinating with the physical fitness laboratory of University, a body composition measure was performed. At the first session, body composition was measured.

During each session, participants were asked to run a standard heating protocol that was led by the coaches of the Trampoline national team for 10 minutes. Before each test, the correct implementation of the error and error criteria and the evaluation of the implementation were explained by the laboratory experts and, if any questions were answered, the items were answered. Participants were allowed to review their implementation practices before performing the main tests. To prevent fatigue, a rest time test was considered sufficiently. Each measurement session was terminated with a standard cooling protocol. Finally, information about the level of trampoline performance at the end of the training camp was received from the technical team members.

\section{Data Analysis Method}

To describe the data, mean, standard deviation, frequency, frequency, and table and graph drawing were used. In the data analysis, firstly, the natural assumption of the distribution of data was investigated using Shapiro Wilk's test. Considering the prediction of the natural distribution of the data, Pearson correlation coefficient was used. All analyzes were performed at 95\% confidence level using Excel software and SPSS version 15. 


\section{Results}

Table 1. Pearson correlation coefficient for determining the relationships between body composition and competitive performance

\begin{tabular}{|c|c|c|c|}
\hline Predictive variables & $\mathrm{N}$ & $\boldsymbol{r}$ & $p$ \\
\hline Height & 45 & 0.152 & 0.342 \\
\hline Body mass & 45 & 0.181 & 0.256 \\
\hline Body mass index & 45 & 0.201 & 0.209 \\
\hline Percentage of water in the body & 45 & 0.021 & 0.895 \\
\hline Body protein percentage & 45 & 0.22 & 0.892 \\
\hline Percent mineralization and mineral content of the body & 45 & -0.194 & 0.224 \\
\hline Body fat percentage & 45 & 0.06 & 0.71 \\
\hline Right-fetal fat percentage & 45 & 0.121 & 0.450 \\
\hline Percentage of liposuction of the right hand & 45 & -0.03 & 0.984 \\
\hline The percentage of fat in the right leg & 45 & 0.138 & 0.389 \\
\hline The percentage of left fetal liposome & 45 & 0.095 & 0.553 \\
\hline Percentage of liposomal liposomal penicillin & 45 & 0.007 & 0.964 \\
\hline Percentage of left foot fat & 45 & 0.111 & 0.491 \\
\hline Body fat percentage & 45 & 0.04 & 0.805 \\
\hline Difference in fat mass of the right and left extremities & 45 & -0.008 & 0.963 \\
\hline Percentage of body muscle & 45 & 0.02 & 0.901 \\
\hline Percentage of right extremity muscle & 45 & 0.005 & 0.974 \\
\hline Percentage of right hand muscle & 45 & 0.118 & 0.461 \\
\hline Percentage of right leg muscle & 45 & -0.038 & 0.816 \\
\hline Left limb muscle mass & 45 & 0.011 & 0.948 \\
\hline Left hand muscle mass & 45 & 0.153 & 0.339 \\
\hline Percentage of left foot muscle & 45 & -0.068 & 0.673 \\
\hline The percentage of trunk muscle & 45 & -0.075 & 0.639 \\
\hline Difference in the percentage of right and left muscles & 45 & -0.059 & 0.714 \\
\hline
\end{tabular}

Table 1 shows that the relationship between the elite male trampolines according to height, body mass, body mass index, body water percentage, protein percentage, soluble mineral content, body fat percentage, right fat percentage, Percentage of fat in right hand, percentage of fat in right leg, left fat percentage, left fat percentage, fat percentage of left foot, trunk fat percentage, difference between left and right fat mass, body mass percentage, percentage of right muscle, right hand muscle, percentage of right muscle, percentage of left muscle, percentage of left leg muscle, trunk muscle percentage and percentage of left and right leg muscles were statistically significant Therefore, it is concluded that there is no significant relationship between body composition and male elite performance (in all cases, $\mathrm{p}<0.05$ ). 
Table 2. Descriptive statistics of physical fitness factors

\begin{tabular}{lrrr}
\hline & Indices & & \\
& $\mathrm{N}$ & \multicolumn{1}{c}{ M } & \multicolumn{1}{c}{ SD } \\
& 45 & 10.97 & 3.96 \\
Shoulder muscle strength (maximum repetition) & 45 & 6.30 & 4.70 \\
Abdominal muscle strength (Maximum repeat) & 45 & 16.55 & 9.38 \\
Muscle endurance (s) & 45 & 51.59 & 13.43 \\
Shoulder joint flexion - upward (\% hand length) & 45 & 74.16 & 7.09 \\
Shoulder Joint Flexibility - Backward (\% Hand Length) & 45 & 14.95 & 4.66 \\
Flexible forward trunk (cm) & 45 & 60.86 & 7.74 \\
Flexural trunk to the back (\% sit tall) & 45 & 42.30 & 5.40 \\
Aerobic power (ml / kg per minute) & 45 & 27.58 & 10.14 \\
Explosive Power - Vertical Jump Record (\% Length) & 45 & 123.85 & 11.25 \\
Explosive Power - Record Jumping Length (\% Length) & 45 & - & - \\
Bleeding the upper muscles of the trunk & 45 & 499.47 & 224.31 \\
Maximum Absolute Bulking Capacity (W) & 45 & 347.90 & 150.37 \\
Absolute Average Bleeding Power (W) & 45 & 194.71 & 102.98 \\
Absolute Power (Watts) & 45 & 9.84 & 2.02 \\
Maximum Relative Expansion Power (watts per kilogram) & 45 & 6.85 & 1.24 \\
Relative mean bleeding power (watts per kilogram) & 45 & 3.78 & 1.32 \\
Minimal Relative Potency (Watts per Kilogram) & 45 & 42.88 & 7.44 \\
Bleeding power of the lower muscles of the trunk (watts per kilogram) & 45 & 60.11 & 8.52 \\
Agility - zigzag test record (repeat in 30 seconds) & 45 & 10.37 & 2.07 \\
Viewing time (watts per kilogram) & 45 & 79.91 & 6.32 \\
Dynamic Balance (\% Foot Length) & 45 & 83.01 & 7.18 \\
Dynamic balance on the right leg (\% foot length) & 45 & 76.82 & 6.44 \\
Dynamic balance on the left foot (\% leg length) & 45 & 6.68 & 4.43 \\
Dynamic balance difference of right and left foot (\% leg length) & & & \\
\hline
\end{tabular}

Table 3. Results of Pearson correlation coefficient to determine the relationship between physical fitness factors and competitive performance

\begin{tabular}{lrrr}
\hline Predictive variables & N & \multicolumn{1}{c}{$\mathrm{R}$} & \multicolumn{1}{c}{$\mathrm{P}$} \\
\hline The muscle strength of the shoulder belt & 45 & 0.294 & $0.05^{*}$ \\
Abdominal muscle strength & 45 & 0.248 & 0.118 \\
Muscle endurance & 45 & -0.029 & 0.868 \\
Shoulder Joint Flexibility - Upward & 45 & 0.162 & 0.312 \\
Shoulder joint flexion - Back to back & 45 & -0.096 & 0.550 \\
Flexural forward trunk & 45 & 0.104 & 0.517 \\
Thoracic torso flexion & 45 & 0.164 & 0.304 \\
Aerobic power & 45 & 0.351 & 0.047 \\
Explosive Power - Vertical Jump Record & 45 & 0.02 & 0.901 \\
Explosive Power - Record Long Jump & 45 & 0.057 & 0.725 \\
Bleeding the muscles of the upper trunk & 45 & - & - \\
Maximum Relative Absolute Power & 45 & 0.057 & 0.731 \\
Medium Relative Bleeding Possibility & 45 & 0.094 & 0.569 \\
Minimal bleeding power & 45 & 0.256 & $0.05{ }^{*}$ \\
Bleeding the muscles of the lower trunk & 45 & 0.207 & 0.194 \\
Agility - Zigzag test record & 45 & 0.011 & 0.947 \\
View Photo Time & 45 & -0.08 & 0.617 \\
Dynamic balance & 45 & 0.082 & 0.613 \\
Dynamic balance on the right foot & 45 & 0.084 & 0.606 \\
Dynamic balance on the left foot & 45 & 0.068 & 0.675 \\
Dynamic balance difference between right and left legs & 45 & 0.017 & 0.915 \\
The muscle strength of the shoulder belt & 45 & 0.284 & $0.05{ }^{*}$ \\
\hline
\end{tabular}

The results of table 3 show that the relationship between the performance of elite male trampolines with muscle strength, shoulder muscle strength, aerobic power, and minimum relative biopsy power is statistically significant, but the relationship between performance with abdominal muscle strength Muscular endurance, shoulder joint flexibility upward, shoulder joint flexibility - backward, forward trunk flexibility, trunk flexural strength, explosive power vertical jump record, explosive power - jump record length, maximum relative tensile strength high tympanic muscle, relative mean muscle tone of upper muscle, trend of lower muscle tone, agility - zigzag test record. The observation image, dynamic balance, dynamic balance on the right foot, dynamic balance on the left leg and the dynamic balance 
difference between the right and left legs are not statistically significant.

Observed relationships indicate that high levels of performance in elite male trampolines are associated with high levels of shoulder muscle strength, aerobic power, and minimal relative healing power. It can be concluded that there is a significant relationship between some of the factors of physical fitness (muscle strength of shoulder belt, aerobic power and minimum relative bias) and the performance of male elite trampolines.

Table 4. Results of regression model of competitive performance level of elite male trampolines

\begin{tabular}{|c|c|c|c|c|c|c|c|c|c|c|c|}
\hline \multirow[t]{4}{*}{$\begin{array}{l}\text { Source } \\
\text { changes }\end{array}$} & of & variables entered in model & df & $\mathrm{R}$ & $\mathrm{R}^{2}$ & $\begin{array}{l}\text { Modified } \\
\mathrm{R}^{2}\end{array}$ & $\boldsymbol{F}$ & $p$ & $R^{2} \Delta$ & FA & $\begin{array}{l}\text { P for } \\
\text { change }\end{array}$ \\
\hline & & $\begin{array}{l}\text { Physical fitness factors 1) Shoulder muscle } \\
\text { strength }\end{array}$ & 3 & 0.31 & 0.096 & 0.023 & 1.31 & 0.284 & 0.096 & 1.31 & 0.284 \\
\hline & & 2) Aerobic power & & & & & & & & & \\
\hline & & 3) Minimal Relative Bonding Power & & & & & & & & & \\
\hline
\end{tabular}

* At level $0.05 \mathrm{p}$

Contradiction results show that the level of competitive performance of elite male trampolines is significant in the strength of shoulder muscle strength, aerobic power, and minimal bleeding ability.

\section{Discussion and Conclusion}

The results of the research on physical fitness factors indicated that the aerobic power, shoulder muscle strength and upper trunk muscle tibial power (minimum relative retardation capacity) were compared to that of trampoline elite male athletes are related and other factors of physical fitness do not have a significant relationship with the performance level of these athletes. These findings suggest that higher aerobic power and higher bleeding power and strength in the upper trunk with higher levels of performance in the trampoline line are associated. However, the trampoline field is considered as an anaerobic field due to the nature of the motion and the duration of its routines, but nevertheless, the importance of aerobic power as a feature of the vastly large volume of infrastructure Athletes' workout and the impact of cardio-pulmonary and circulatory systems in the recycling process can be considered.

Similar findings have also been reported on the importance of aerobic power in the field of gymnastics. For example, Douda et al. (2008) reported a relationship between the aerobic power and the performance of artistic gymnasts $(r=0.49)$, which is expected to be comparable with the trampoline's motion (less time to run). There is a weaker relation between aerobic power and performance in this field than in the field of gymnastics. The findings of this study also indicate the existence of such a relationship $(\mathrm{r}=0.351)$.

On the other hand, the meaningful relationship between shoulder muscle strength and the high muscle strength of the upper trunk with the level of function emphasizes the importance of the role of the upper muscles of the trunk in creating the momentum needed to run the skills of the trampoline, because the skill those with a higher degree of difficulty in this field require the forward and backward rotation, which requires high muscular strength and muscle strength in the upper trunk. Although the existence of such meaningful relationships, in part, determines the factors involved in the exercise performance in the trampoline field, the predictive power of these variables should be confirmed on the basis of regression analysis.

In this regard, the results of regression analysis of the level of competitive performance based on the selected factors of physical fitness (muscle strength of shoulder belt, aerobic power and minimum relative power of biosynthesis) showed that these factors cannot predict the level of performance and only $9.6 \%$ of the performance of elite male trampolines is related to these variables. Douda et al. (2008) reported that the contribution of the flexibility factor to gymnastics performance was $12.1 \%$, explosive power $9.2 \%$, aerobic power $4.7 \%$, and fuel metabolism $4.6 \%$, which was does not match the trampoline. The reason for the inconsistency may be related to the difference in the nature of the two competitive disciplines and the gender difference and the competitive level of the samples. In general, according to the findings of the present study about the factors of physical fitness, only the factors of aerobic capacity, strength and power of the muscles of the upper trunk were related to the performance of elite male tricolor.

Findings of the study on the composition of the body of elite male trampolines showed that none of the body composition indices was significant with the performance of these athletes. Considering that in many studies, especially in the field of gymnastics (for example, Irratha Amigo et al., 2009; Di Kagno et al., 2009; New Age, 2011), there are significant correlations between body composition and exercise performance indices, therefore it seems that the lack of correlation between these variables is related to the characteristics of the samples studied in this study.

For example, most of the research samples are a combination of athletes with different levels of stress and, consequently, a different body composition that this strategy facilitates in sampling the ability to discover relationships between body composition indices and athletic performance. But in the present study, the sample was all from the athletes present in the Trampoline team, which, in terms of body composition, were more homogeneous than those of other studies. 
Gomez-Landro et al. (2010) found that Spanish elite female gymnasts over 15 years old had $13.37 \%$ fat and mesomorph-endomorph body mass, but under the age of 15 years had body mass of $11.18 \%$ and a balanced physical type. However, in the present study, the percentages of fatty male elite male trampolines for the age group under 15 years old and over 15 years old were $12.13 \pm 4.20$ and $11.02 \pm 4.18$, respectively, do not match with findings of Gomez-Landro et al. (2010) about the elite Spanish female trampolines the discrepancy between these two studies can be attributed largely to the gender and developmental differences between men and women, because with the increase in age, women share a larger proportion of fat mass (Heywood, 2006). .In the studies in gymnastics, body composition indices were important determinants of athletic performance. For example, Nowzuhuri (2011) found that elderly male gymnasts had lower fat percentages with higher weight and higher body mass index (high muscle and low fat) with higher competitive performance. Similar findings were found in other studies in the field of gymnastics by Faria and Faria (1989), Grantham (2000), Dashti (2016), Giorpopoulos et al. (2004), Taherkhani (2006), Rodriguez and Bral De La Rosa (2006), Irrata Amigo et al. (2009), and Di Cagno et al. (2008).

However, Gomez-Landro et al. (2010), by comparing their findings with gymnasts reference data, emphasized that trampolines' athletes had a different type of artistic gymnasts, such that trompulsins in the body type has a lower ectomorphic component. Accordingly, matching performance predictors in the field of trampolines and gymnastics may be questionable. However, due to the limited information available in the trampoline field, definite comments on the pattern of association between body composition indices and the performance of elite trampolines are difficult, but according to the findings of the present study, we can conclude there is no relation between the body composition and the performance of male trampolines.

\section{References}

Dashti, K., \& Mohammad, H. (2016). Relationship between some anthropometric characteristics and physical fitness factors with the performance of gymnasts in the superior male boys. Master's thesis, University of Guilan, Faculty of Physical Education and Sport Sciences.

Di Cagno, A., Baldari, C., Battaglia, C., Guidetti, L. I., \& Piazza, M. (2008). Anthropometric characteristics evolution in elite rhythmic gymnasts. Italian Journal of Anatomy and Embryology, 113(1), 29-35.

Douda, H. T., Toubekis, A. G., Avloniti A. A., \& Tokmakidi, S. P. (2008). Physiological and anthropometric determinants of rhythmic gymnastics performance. International Journal of Sport Physiology and Performance, 3, 41-45. https://doi.org/10.1123/ijspp.3.1.41

Dzhafarov, M. A., \& Vasil'chuk, A. L. (1987). Anthropometric characteristics of highly qualified female gymnasts. Arkhiv Anatomii Gistologii I Embriologii, 93(8), 33-37.

Faria, I. E., \& Faria, E. W. (1989). Relationship of the anthropometric and physical characteristics of male junior gymnasts to performance. J Sports Med Phys Fitness, 29(4), 369-378.

Georgopoulos, N. A., Theodoropoulou, A., Leglise, M., Vagenakis, A. G., \& Markou, K. B. (2004). Growth and skeletal maturation in male and female artistic gymnasts. The Journal of Clinical Endocrinology \& Metabolism, 89(9), 4377-4382. https://doi.org/10.1210/jc.2003-031864

Gómez-Landero, L. A., Vernetta, M., \& López-Bedoya, J. (2010). Somatotype and body composition in elite Spanish female trampolinist. International Journal of Sport Science, 19(6), 141-153.

Grandjean, B. D., Taylor, P. A., \& Weiner, J. (2002). Confidence, concentration, and competitive performance of elite athletes: a natural experiment in Olympic gymnastics. Journal of Sport \& Exercise Psychology, 24, 320-327. https://doi.org/10.1123/jsep.24.3.320

Grantham, N. J. (2000). Body composition and physiological characteristics of male and female national and international high performance gymnasts. Journal of Sports Sciences, 18(1), 24-35.

Hume, P. A., Hopkins, W. G., Robinson, D. M., Robinson, S. M., \& Hollings, S. C. (2014). Predictors of attainment in rhythmic sportive gymnastics. J Sports Med Phys Fitness, 33(4), 367-377.

Irurtia Amigo, A., Busquets Faciaben, A., Marina Evrard, M., Galilea Ballarini, P. A., \& Carrasco Marginet, M. (2009). Height, weight, somatotype and body composition in elite Spanish gymnasts from childhood to adulthood. Apunts Med Esport, 161, 18-28.

Lindner, K. J., Caine, D. J., \& Johns, D. P. (1991). Withdrawal predictors among physical and performance characteristics of female competitive gymnasts. J. Sports Sci., 9(3), 259-272. https://doi.org/10.1080/02640419108729888

Macmillan Reference USA (2007). Prescription Medications and Athletic Performance. World of Sports Science, 
Retrieved February 03, 2012 from HighBeam Research: http://www.highbeam.com/doc/1G2-3451100415.html

Rodriguez, B. E., \& Berral de la Rosa, F. J. (2006). Morfhological assesment in elite argentineans male gymnasts. Rev Bras Cineantropom Desempenho Hum, 8(4), 16-24.

Sherman, R. T., Thompson, R. A., \& Rose, J. (1996). Body mass index and athletic performance in elite female gymnasts. Journal of Sport Behavior, 19, 338-346.

TaherKhani, A. (2011). The prediction of elite male gymnasts based on self-efficacy, psychological skills and some anthropometric features. Master's Thesis, Shiraz University.

TaherKhani, A. (2011). The prediction of elite male gymnasts based on self-efficacy, psychological skills and some anthropometric features. Master's Thesis, Shiraz University.

Vandorpe, B., Vandendriessche, J., Vaeyens, R., Pion, J., Lefevre, J., Philippaerts, R., \& Lenoir, M. (2011). Factors Discriminating Gymnasts by Competitive Level. Int. J. Sports Med., 32(8), 591-597. https://doi.org/10.1055/s-0031-1275300

Zuniga, J., Housh, T. J., Camic, C., \& et al. (2011). Yearly changes in the anthropometric dimensions of female high school gymnasts. Journal of Strength \& Conditioning Research, 25(1), 124-128.

https://doi.org/10.1519/JSC.0b013e3181bb0d92

\section{Copyrights}

Copyright for this article is retained by the author(s), with first publication rights granted to the journal.

This is an open-access article distributed under the terms and conditions of the Creative Commons Attribution license which permits unrestricted use, distribution, and reproduction in any medium, provided the original work is properly cited. 